\title{
Genome sequencing accuracy by RCA-seq versus long PCR template cloning and sequencing in identification of human papillomavirus type 58
}

\author{
Xiaohong Wang ${ }^{1}$, Yang $\mathrm{Li}^{1,2}$, Ting $\mathrm{Ni}^{3,4}$, Xing $\mathrm{Xie}^{2}$, Jun Zhu ${ }^{3}$ and Zhi-Ming Zheng ${ }^{1 *}$
}

\begin{abstract}
Background: Genome variations in human papillomaviruses (HPVs) are common and have been widely investigated in the past two decades. HPV genotyping depends on the finding of the viral genome variations in the L1 ORF. Other parts of the viral genome variations have also been implicated as a possible genetic factor in viral pathogenesis and/or oncogenicity.

Results: In this study, the HPV58 genome in cervical lesions was completely sequenced both by rolling-circle amplification of total cell DNA and deep sequencing (RCA-seq) and by long PCR template cloning and sequencing. By comparison of three HPV58 genome sequences decoded from three clinical samples to reference HPV-58, we demonstrated that RCA-seq is much more accurate than long-PCR template cloning and sequencing in decoding HPV58 genome. Three HPV58 genomes decoded by RCA-seq displayed a total of 52 nucleotide substitutions from reference HPV58, which could be verified by long PCR template cloning and sequencing. However, the long PCR template cloning and sequencing led to additional nucleotide substitutions, insertions, and deletions from an authentic HPV58 genome in a clinical sample, which vary from one cloned sequence to another. Because the inherited error-prone nature of Tgo DNA polymerase used in preparation of the long PCR templates of HPV58 genome from the clinical samples, the measurable error rate in incorporation of nucleotide into an elongating DNA template was about $0.149 \% \pm 0.038 \%$ in our studies.
\end{abstract}

Conclusions: Since PCR template cloning and sequencing is widely used in identification of single nucleotide polymorphism (SNP), our data indicate that a serious caution should be taken in finding of true SNPs in various genetic studies.

Keywords: Human papillomaviruses, HPV58, Cervical cancer, Single nucleotide polymorphism, Genotyping, Genome variations, Rolling circle amplification, DNA deep sequencing

\section{Introduction}

Human papillomaviruses (HPVs) are a group of more than 200 genotypes of small DNA tumor viruses (http://pave.niaid.nih.gov/\#home) and can be grouped clinically as high-risk (oncogenic) types, which are frequently associated with invasive cervical cancer, and low-risk (non-oncogenic) types, which are found mainly in genital warts. To date, fifteen HPV types has been classified as high-risk HPVs, including HPV16,

\footnotetext{
* Correspondence: zhengt@exchange.nih.gov

'Tumor Virus RNA Biology Section, Gene Regulation and Chromosome Biology Laboratory, Center for Cancer Research, National Cancer Institute, National Institutes of Health, Bethesda, MD, USA

Full list of author information is available at the end of the article
}

$18,31,33,35,39,45,51,52,56,58,59,68,73$, and 82 $[1,2]$. Among the high-risk HPVs, HPV16 and HPV18 are the principal causes of cervical cancer, with a combined, worldwide contribution to $\sim 70 \%$ of invasive cervical cancer $[3,4]$. HPV58 has been found to be more prevalent than HPV18 in cervical intraepithelial neoplasia (CIN) lesions and appears almost equal frequency as HPV18 in cervical cancers in Zhejiang province, China [5] and other Asian countries [6,7]. We recently characterized HPV58 genome variations and RNA expression in women with cervical lesions [8].

HPV genome in size of $\sim 7.9 \mathrm{~kb}$ encodes eight open reading frames (ORFs, E6, E7, E1, E2, E4, E5, L2 and L1) from one strand of its double stranded, circular genome

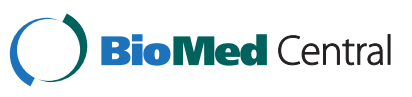


in one direction and a long control region (LCR) between L1 and E6 [9]. The classification of papillomaviruses by genotyping depends on the most conserved L1 ORF. A new type of papillomavirus is granted when its DNA sequence of L1 ORF differs by more than $10 \%$ from the closest known HPV type. A subtype indicates the difference between $2 \%$ and $10 \%$ and a variant represents less than $2 \%$ difference [10-12]. Nucleotide variations in the LCR region are often used to describe intratype diversity or variant lineage [12-15]. Despite that HPV genome is viewed as a stable genome, its genome sequence variations in a given genotype appear different from one laboratory to another and from one geological region to another [14-22]. The reported variations have been found not only in the L1 ORF and the LCR region, but also in other parts of the viral genome and were identified mostly, if not all, by PCR amplicon sequencing. Although it is always questionable whether an authentic variation (s) does exist in the reported HPV genotype because of the use of error-prone Taq polymerase in PCR amplification, many attempts have been made to correlate such genome variations to HPV pathogenesis and viral carcinogenicity [23-26]. Thus, a more reliable method is urgently needed to study HPV genome variation and its possible biological role in HPV infection. Recently, next-generation sequencing (NGS) techniques are emerging as a promising application for HPV genotyping and identification of rare single nucleotide polymorphism (SNP) [8,27-30].

In this report, we analyzed and compared three HPV58 genome sequences from three cervical lesion samples decoded by an RCA-seq (rolling-circle amplification and deep sequencing) $[8,31]$, a technique that does not require prior knowledge of the underlying genome, and by long PCR template cloning and Sanger sequencing. We demonstrated that RCA-seq is more reliable method in identification of an authentic nucleotide variation in an episomal viral genome.

\section{Results}

To analyze single nucleotide polymorphism (SNP) in HPV genome, RCA was applied to enrich copy number of an episomal HPV58 genome from each of three cervical lesion samples. To quantify HPV58 and host GAPDH copy numbers from the same sample before and after RCA, a quantitative real-time PCR (qPCR) was performed on $\simeq 100 \mathrm{pg}$ of sample DNA (samples 10 and 13). The threshold cycle $(\mathrm{Ct})$ values from 2 repeats were calculated for copy number analysis. Human GAPDH DNA in linear form served as an internal control. As expected, we found that RCA enriched HPV58 genome copy number in the sample 13 from 1154 copies to 7317908 copies (more than 6300-fold enrichment) while displaying no enrichment activity to host
GAPDH linear DNA. The Ct values for GAPDH DNA were 23.42 before RCA enrichment and 22.76 after RCA enrichment in the sample 13. A similar result was observed in the sample 10, in which HPV58 genome copy was under detection level before RCA, but could be enriched by RCA to 20481 copies quantified by qPCR (Figure 1A) and became detectable by agarose gel electrophoresis (Figure 1B), with only a little enrichment of host GAPDH linear DNA.

To have the RCA products being deep-sequenced using an Illumina HiSeq-2000 platform, we subsequently debranched the RCA products and prepared a paired-end library from each RCA sample [8,31]. More than 104 millions of paired-end reads from the sample 9, 161 millions from the sample 10, and 120 millions from the sample 13 were obtained using the RCA-seq. Among those reads, 3453 in the sample 9, 13249 in the sample 10 , and 1.4 million in the sample 13 could be mapped to the reference HPV58 genome (GI: 222386) [32], giving a complete coverage of a full-length HPV58 genome [8]. Detailed analyses of the nucleotide sequence at each position against the reference HPV58 genome [32] showed that the HPV 58 genomes in three clinical samples contain nucleotide substitutions being verifiable either in other samples or in the same sample by the second RCA-seq [8].

We subsquently cloned the full-length, episomal HPV58 genome from each clinical sample and compared each genome sequence obtained by primer-walking Sanger sequencing to the genome sequence obtained by the RCA-seq. To do so, the same RCA product from each clinical sample prepared for RCA-seq, without S1 digestion and DNA shearing, was linearized by AgeI digestion at nt 62 position or by DraII digestion at nt 4536 position of HPV58 genome. Subsequently, two large fragments, a $\simeq 3.5-\mathrm{kb}$ fragment from nt 3506 to 7036 of HPV58 genome linearized from AgeI-digested RCA product and a $\simeq 4.6$-kb fragment from nt 6905 to 3694 of HPV58 genome linearized from DraII-digested RCA product from each clinical sample, were amplified separately by high-fidelity thermostable Tgo DNA polymerase with proofreading activity and inserted separately into a pCR-XL-TOPO vector. Two clones for each fragment and four clones for each RCA product of the individual clinical sample were obtained (Table 1). All plasmid clones were then sequenced by conventional primer-walking Sanger sequencing on both strands and the full-length viral genome sequence was aligned against the reference HPV58 genome and its corresponding HPV58 genome as determined by RCA-seq.

As shown in Figure 2, 22 nucleotide substitutions in the sample 9-derived HPV58 (CNZJ-3, GenBank accession number KC860270), 39 in the sample 10-derived HPV58 (CNZJ-2, GenBank accession number KC860271), and 37 


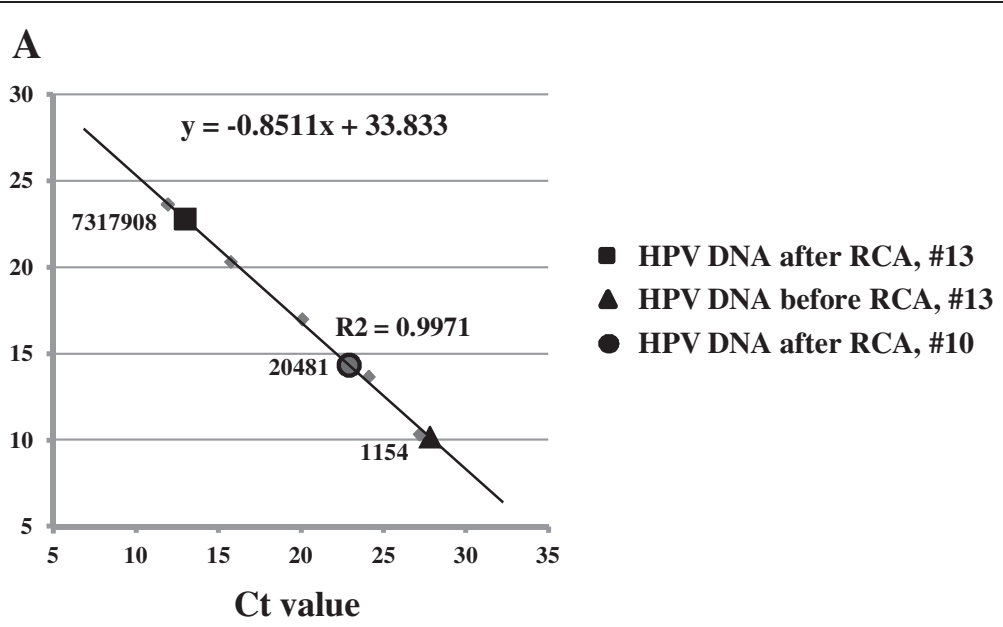

B

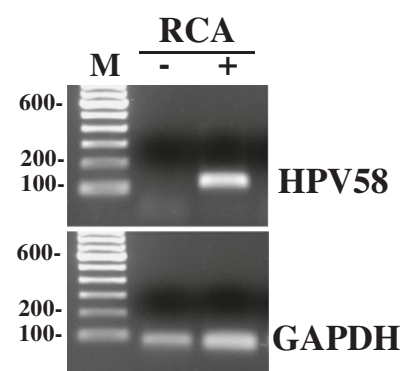

Figure 1 Enrichment of HPV genomic DNA by RCA from cervical samples. (A) HPV58 genome copy numbers before and after RCA enrichment. Real-time PCR (qPCR) was performed with an HPV58-specific primer pair on 100 pg of sample DNA (sample 10 and sample 13) either before or after RCA enrichment. A 10-fold serial dilution, starting from $100 \mathrm{pg}\left(\sim 1.3 \times 10^{7}\right.$ copies $)$ of the plasmid pXW59-1 which contains an HPV58 DNA fragment from nt 6906 to 3695 was amplified using the same primer set by qPCR to create a standard curve. The threshold cycle (Ct) values of qPCR data from 2 repeats were calculated for copy number analysis. GAPDH was used as an internal control. (B) HPV58 DNA in the sample 10 was under detection level before RCA, but became detectable by agarose gel electrophoresis after enrichment by RCA to 20481 copies as quantified by $q P C R$.

in the sample 13-derived HPV58 (CNZJ-1, GenBank accession number KC860269) found in RCA-seq [8] were all verified in the HPV58 plasmid clones derived from individual clinical samples, but the long PCR template cloning and sequencing created additional nucleotide variations to its corresponding viral genome sequence determined by RCA-seq. The sample 9-derived plasmid sequence-1 (seq-1) decoded from pXHW54-1 plus pXHW57-1 contained additional twelve nucleotide substitutions and four nucleotide insertions and its seq-2 from pXHW54-2 plus pXHW57-2 contains five separate nucleotide substitutions, two separate nucleotide insertions, and one nucleotide deletion (Figure 2 and Table 2). For the sample 10-derived plasmid seq-1 decoded from pXHW55-1 plus pXHW58-1 and the seq-2 from pXHW55-2 plus pXHW58-2, the additional nucleotide variations include five substitutions, two insertions, and one deletion in its seq- 1 and ten separate substitutions in its seq-2 (Figure 2 and Table 2). Similarly, the viral genome sequence derived from the sample 13 displayed additional twelve nucleotide substitutions and two nucleotide insertions in its seq- 1 decoded from pXHW56-1 plus pXHW59-1 and nine separate substitutions and one separate insertion in its seq-2 decoded from pXHW56-2 plus pXHW59-2 (Figure 2 and Table 2). Further analyses of the additional variations generated by the long PCR template cloning and sequencing showed that some of them could create or inactivate a restriction enzyme cutting site as summarized in Table 3 and could be therefore verified by the restriction enzyme digestion. As shown in Figure 3, AfeI, EcoRV, or FspI could digest the PCR products, respectively, from pXHW57-1, pXHW55-1, or pXHW59-1, but not the PCR products from their corresponding RCA preps derived from the same clinical sample, indicating that these nucleotide variations do not exist in the authentic HPV58 genome, rather they were most likely introduced by the long PCR template cloning and sequencing.

Notably, these additional nucleotide variations from the long PCR template cloning and sequencing were random and not duplicable each other between two cloned viral genome sequences derived from the same clinical samples. In addition, we found that the insertions 
Table 1 The list of plasmids constructed and used in the study

\begin{tabular}{ll}
\hline Plasmid & Characteristics \\
\hline pXHW54 & $\begin{array}{l}\text { RCA product from HPV58 CNZJ-3 (sample 9) was amplified } \\
\text { with Pr3506 (OYL35) and Pr7036 (OXHW262), gel purified and } \\
\text { cloned into pCR-XL-TOPO vector. Insertion was verified by } \\
\text { sequencing. }\end{array}$ \\
pXHW55 & $\begin{array}{l}\text { RCA product from HPV58 CNZJ-2 (sample 10) was amplified } \\
\text { with Pr3506 (oYL35) and Pr7036 (oXHW262), gel purified and } \\
\text { cloned into pCR-XL-TOPO vector. Insertion was verified by } \\
\text { sequencing. }\end{array}$
\end{tabular}

pXHW56 RCA product from HPV58 CNZJ-1 (sample 13) was amplified with Pr3506 (oYL35) and Pr7036 (oXHW262), gel purified and cloned into PCR-XL-TOPO vector. Insertion was verified by sequencing.

pXHW57 RCA product from HPV58 CNZJ-3 (sample 9) was amplified with Pr6906 (oXHW263) and Pr3694 (oXHW264), gel purified and cloned into PCR-XL-TOPO vector. Insertion was verified by sequencing.

pXHW58 RCA product from HPV58 CNZJ-2 (sample 10) was amplified with Pr6906 (oXHW263) and Pr3694 (oXHW264), gel purified and cloned into PCR-XL-TOPO vector. Insertion was verified by sequencing.

pXHW59 RCA product from HPV58 CNZJ-1 (sample 13) was amplified with Pr6906 (oXHW263) and Pr3694 (oXHW264), gel purified and cloned into PCR-XL-TOPO vector. Insertion was verified by sequencing.

Two bacterial colonies were picked up for each plasmid preparation and the obtained plasmid preps were named as a plasmid-1 or -2 (such as pXHW54-1 or -2 described in the text).

or deletions identified from the cloned HPV58 genome fragment always happened at a run of multiple A, T, or C. Together, a total of additional 70 nucleotide variations were identified from six cloned HPV58 genome sequences which differ from the RCA-seq-defined HPV58 genome sequences. We concluded that these variations were derived from long PCR template amplification with a less efficient proof-reading Tgo DNA polymerase. By calculation, the Tgo DNA polymerase exhibited an error rate of $\sim 0.149 \% \pm 0.038 \%$ in our long PCR template preparation per HPV58 genome in size of $7824 \mathrm{nts}$.

\section{Discussion}

Rolling-circle amplification (RCA) has been developed as a powerful tool to amplify a whole genome in an episomal form for microbial genome organization and phylogenetic analyses [33]. In this study, HPV58 genome in cervical lesions was decoded, respectively, by RCA-seq and by long PCR template cloning and sequencing. We demonstrated that the RCA-seq is better than the long PCR template cloning and sequencing in providing more accurate HPV58 genome sequence. This conclusion was drawn by comparing the same genome sequence decoded by the two techniques. All nucleotide variations to the reference HPV58 genome identified from each clinical sample by RCA-seq could be verified by the long PCR template cloning and sequencing, but not vice versa. In addition, the long PCR template cloning and sequencing technique creates additional sequence variations, which were random and not verifiable from one cloned sequence to another derived from the same sample.

Although the viral DNA from each clinical sample was enriched by RCA using phi29 DNA polymerase [34,35] which features only a minimal error rate of 1 in $10^{6}-10^{7}$ [36] and the reported sequencing accuracy for a NGS with Illumina HiSeq platform is $98 \%$ [37], we found that RCA-seq in this report displayed a more reliable approach, by calling consensus bases, in decoding the entire HPV58 genome in size of 7824 nts. This could be achieved by using a combination of both high fidelity phi29 DNA polymerase at the experimental side and appropriate sequencing data analysis at the computational side. First, we defined a position as identical if more than $95 \%$ of the bases obtained were identical to those of the reference genome. Second, nucleotide substitutions were identified if the majority of bases in the reads differ from the reference genome with read depth of 5 or more. Third, nucleotide positions with read depth less than 5 were treated as ambiguous sites since there is insufficient depth to make a high confidence call [8].

We determined that additional nucleotide variations observed in our long PCR template cloning and sequencing were derived from the proofreading error of Tgo DNA polymerase used in our long PCR template preparation. Although the enriched DNA chimeras in the RCA reaction which might carry a misincorporated base from phi29 DNA polymerase amplification were served, after debranching by AgeI- or Drall-specific digestion of HPV58 genome, for subsequent long PCR template preparation and cloning, this carry-over possibility appears to be unlikely because the phi29 DNA polymerase features only a minimal error rate of 1 in $10^{6}-10^{7}$ [36] in contrast to $\simeq 3$ in $10^{4}$ for PCR with Taq DNA polymerase [38]. Other studies indicate that the majority of DNA sequence changes introduced during PCR are polymerase-mediated and PCR accumulates about one mutation per 400 bases after 30 cycles $[38,39]$. The PCR-induced transitions are the major source of error in other NGS studies $[40,41]$. The reported Tgo DNA polymerase from the Roche product manual has an overall error frequency of $\sim 0.2 \%$ in nucleotide misincorporation during PCR amplification, very close to what we found in our study.

In summary, a more accurate RCA-seq superior to PCR cloning and sequencing has been developed in our lab for HPV genotyping and determination of SNPs in clinical HPV variants. Our protocol provides much higher numbers of specific HPV reads [8] over other high-throughput NGS platforms/protocols applied to HPV genotyping [27-30]. Since PCR sequencing and PCR template cloning and sequencing are widely used in various epidemiology 
A

\begin{tabular}{ccccc} 
& & \multicolumn{3}{c}{ Sample } \\
\cline { 2 - 5 } NT & Ref & RCA & S1 & S2 \\
\hline $\mathbf{8 1}$ & G & C & C & C \\
$\mathbf{3 8 8}$ & A & C & C & C \\
744 & T & G & G & G \\
$\mathbf{8 0 3}$ & T & C & C & C \\
1167 & T & & C & \\
$\mathbf{1 1 9 4}$ & T & C & C & C \\
$\mathbf{1 4 5 3}$ & C & T & T & T \\
$\mathbf{1 5 0 3}$ & T & C & C & C \\
2354 & T & & & C \\
2494 & A & & G & \\
2925 & T & & C & \\
3137 & G & & A & \\
3217 & A & & G & \\
$\mathbf{3 4 3 0}$ & A & G & G & G \\
$\mathbf{3 5 5 0}$ & T & C & C & C \\
$\mathbf{3 5 8 7}$ & T & G & G & G \\
$\mathbf{3 5 9 6}$ & G & T & T & T \\
$\mathbf{3 6 6 2}$ & T & C & C & C \\
3940 & T & & C & \\
$\mathbf{3 9 7 8}$ & A & C & C & C \\
$\mathbf{4 0 2 9}$ & G & T & T & T \\
$\mathbf{4 0 3 3}$ & C & T & T & T \\
$\mathbf{4 6 2 1}$ & A & C & C & C \\
$\mathbf{4 6 2 7}$ & T & C & C & C \\
4997 & T & & & C \\
$\mathbf{5 3 9 5}$ & G & C & C & C \\
$\mathbf{5 4 8 9}$ & G & A & A & A \\
$\mathbf{5 5 5 6}$ & T & & G & \\
6073 & A & & & G \\
$\mathbf{6 2 7 9}$ & T & & C & \\
6352 & T & & C & \\
$\mathbf{6 5 1 6}$ & T & & C & \\
$\mathbf{6 5 6 0}$ & A & G & G & G \\
$\mathbf{6 6 8 8}$ & C & A & A & A \\
7327 & G & & & A \\
7451 & G & & A & \\
7679 & T & & C & \\
7789 & G & & & A \\
7793 & A & G & G & G \\
Total & & $\mathbf{2 2}$ & $\mathbf{3 4}$ & $\mathbf{2 7}$ \\
\hline & & & & \\
& & &
\end{tabular}

B

\begin{tabular}{ccccc} 
& & \multicolumn{3}{c}{ Sample 10 } \\
\cline { 3 - 6 } NT & Ref & RCA & S1 & S2 \\
\hline $\mathbf{3 0 7}$ & C & T & T & T \\
$\mathbf{6 6 7}$ & T & & & A \\
$\mathbf{6 9 4}$ & G & A & A & A \\
$\mathbf{7 4 4}$ & T & G & G & G \\
$\mathbf{7 6 1}$ & G & A & A & A \\
$\mathbf{9 4 8}$ & G & A & A & A \\
$\mathbf{1 1 9 4}$ & T & C & C & C \\
$\mathbf{1 3 6 0}$ & G & C & C & C \\
$\mathbf{1 4 5 3}$ & C & T & T & T \\
1462 & A & & & G \\
$\mathbf{1 7 8 2}$ & C & T & T & T \\
1895 & T & & & C \\
2289 & T & & C &
\end{tabular}

$2289 \mathrm{~T}$

$2340 \quad \mathrm{~T}$

2935 A

$3145 \mathrm{~T}$

$3445 \mathrm{C}$

3550 T

3587

$\begin{array}{ll}3596 & \text { G } \\ 3824 & \text { T }\end{array}$

3949 T

$\begin{array}{ll}3949 & \mathrm{~T} \\ 3988 & \mathrm{~T}\end{array}$

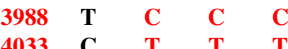

4136 C A A A

4255 A

$4295 \quad \mathrm{~T}$

4408 A

$\begin{array}{lllllllll}4627 & \mathrm{~T} & \mathrm{C} & \mathrm{C} & \mathrm{C}\end{array}$

4945 A G G G

$5095 \mathrm{~T}$

5104 A

5119 A

5143 T

5266 G

$5378 \quad \mathrm{C}$

T T T

$\begin{array}{lllll}5437 & \text { A } & \text { G } & \text { G } & \text { G }\end{array}$

5489 G $\quad$ A $\quad$ A $\quad$ A

5540 A $\quad$ C $\quad$ C $\quad$ C

6014 A $C$ C $\quad$ C

6074 C

$\begin{array}{lllll}6275 & \text { T } & & \text { C } \\ 6416 & \text { I } & \text { G } & \text { G } & \text { G }\end{array}$

$\begin{array}{lllll}6416 & \text { A } & \text { G } & \text { G } & \text { G } \\ 6434 & \text { T } & \text { C } & \text { C } & \text { C }\end{array}$

$\begin{array}{lllll}6539 & \text { A } & \text { G } & \text { G } & \text { G }\end{array}$

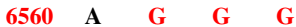

$\begin{array}{lllll}6641 & \text { G } & \text { A } & \text { A } & \text { A } \\ 6827 & \text { C } & \text { T } & \text { T } & \text { T }\end{array}$

$6855 \mathrm{~T} \quad \mathrm{C}$

6994 A G

7039 A G

$\begin{array}{lllll}7266 & \text { C } & \text { T } & \text { T } & \text { T } \\ 7714 & \text { A } & \text { G } & \text { G } & \text { G }\end{array}$

\begin{tabular}{llll} 
Total & 39 & 44 & 49 \\
\hline
\end{tabular}
C

\begin{tabular}{|c|c|c|c|c|}
\hline \multirow{2}{*}{ NT } & \multirow[b]{2}{*}{ Ref } & \multicolumn{3}{|c|}{ Sample 13} \\
\hline & & $\mathrm{RCA}$ & $\mathrm{S} 1$ & $\mathrm{~S} 2$ \\
\hline 307 & $\mathbf{C}$ & $T$ & $\bar{T}$ & $T$ \\
\hline 680 & A & & & G \\
\hline 694 & G & A & A & A \\
\hline 744 & $\mathbf{T}$ & G & G & G \\
\hline 761 & G & A & $\mathbf{A}$ & A \\
\hline 948 & G & A & A & A \\
\hline 1194 & $\mathbf{T}$ & C & C & C \\
\hline 1217 & A & & & $\mathrm{T}$ \\
\hline 1360 & G & C & $\mathrm{C}$ & $\mathrm{C}$ \\
\hline 1453 & $\mathrm{C}$ & $\mathbf{T}$ & $\mathbf{T}$ & $\mathbf{T}$ \\
\hline 1782 & $\mathrm{C}$ & $\mathbf{T}$ & $\mathbf{T}$ & $\mathbf{T}$ \\
\hline 2340 & $\mathbf{T}$ & $\mathrm{C}$ & $\mathrm{C}$ & $\mathrm{C}$ \\
\hline 2346 & A & & G & \\
\hline 2721 & A & & G & \\
\hline 2784 & $\mathrm{~T}$ & & G & \\
\hline 2860 & A & & G & \\
\hline 2935 & A & $\mathrm{C}$ & $\mathrm{C}$ & $\mathrm{C}$ \\
\hline 3245 & A & & & G \\
\hline 3445 & C & G & G & G \\
\hline 3550 & $\mathbf{T}$ & $\mathrm{C}$ & C & $\mathrm{C}$ \\
\hline 3587 & $\mathbf{T}$ & G & G & G \\
\hline 3596 & G & $\mathbf{T}$ & $\mathbf{T}$ & $\mathbf{T}$ \\
\hline 3915 & $\mathrm{~T}$ & & & $\mathrm{C}$ \\
\hline 3949 & $\mathbf{T}$ & $\mathrm{C}$ & $\mathrm{C}$ & $\mathrm{C}$ \\
\hline 3988 & $\mathbf{T}$ & C & C & C \\
\hline 4033 & $\mathrm{C}$ & $\mathbf{T}$ & $\mathbf{T}$ & $\mathbf{T}$ \\
\hline 4136 & C & A & A & A \\
\hline 4465 & $\mathrm{~T}$ & & $\mathrm{C}$ & \\
\hline 4627 & $T$ & $\mathrm{C}$ & $\mathrm{C}$ & $\mathrm{C}$ \\
\hline 4735 & $\mathrm{~T}$ & & $\mathrm{C}$ & \\
\hline 4885 & A & & G & \\
\hline 4945 & A & G & G & G \\
\hline 5104 & $\mathbf{A}$ & G & G & G \\
\hline 5119 & A & G & G & G \\
\hline 5143 & $\mathbf{T}$ & G & G & G \\
\hline 5193 & A & & G & \\
\hline 5266 & G & A & A & A \\
\hline 5302 & $\mathrm{~T}$ & & & $\mathrm{C}$ \\
\hline 5375 & A & G & G & G \\
\hline 5395 & G & C & C & C \\
\hline 5489 & G & A & A & A \\
\hline 5540 & $\mathbf{A}$ & $\mathrm{C}$ & $\mathrm{C}$ & C \\
\hline 5687 & $\mathrm{~T}$ & & $\mathrm{C}$ & \\
\hline 5749 & A & & G & \\
\hline 5871 & $\mathrm{C}$ & & & $\mathrm{T}$ \\
\hline 6014 & $\mathbf{A}$ & C & C & C \\
\hline 6287 & $\mathrm{~T}$ & & $\mathrm{C}$ & \\
\hline 6330 & A & & & G \\
\hline 6416 & $\mathbf{A}$ & G & G & G \\
\hline 6434 & $\mathbf{T}$ & $\mathrm{C}$ & C & C \\
\hline 6539 & $\mathbf{A}$ & G & G & G \\
\hline 6641 & G & A & A & $\mathbf{A}$ \\
\hline 6870 & $\mathrm{~T}$ & & $\mathrm{C}$ & \\
\hline 6950 & G & A & $\mathbf{A}$ & A \\
\hline 6975 & $\mathrm{~T}$ & & & C \\
\hline 7266 & C & $\mathrm{T}$ & $T$ & $\mathbf{T}$ \\
\hline 7714 & A & G & G & G \\
\hline 7791 & $\mathrm{~T}$ & & & $\mathrm{C}$ \\
\hline Total & & 37 & 49 & 46 \\
\hline
\end{tabular}

Figure 2 Nucleotide substitutions identified in HPV58 isolates to the reference HPV58 [32] by RCA-seq and by cloning sequencing. RCA-seq results were compared to the sequencing results of two individual bacterial clones of each plasmid for samples 9 (A), 10 (B), and 13 (C). S1 and S2 denotes Sanger sequence \#1 (clone \#1) and \#2 (clone \#2), respectively. Common nucleotide substitutions at positions in the reference HPV58 genome seen from RCA-seq to cloning sequencing were colored in red. The nucleotide substitutions identified only by cloning sequencing were shown in black.

studies to identify natural variants of an HPV type, caution should be taken in evaluation of any new SNPs found by these assays as PCR amplification might introduce nucleotide misincorporation into the studying genotype (s).

\section{Materials and methods} Sample DNA preparation

Three HPV58- positive cervical CIN2/3 tissues were collected from Women's Hospital, School of Medicine, 
Table 2 Nucleotide insertion and deletion created by long-PCR amplification in HPV58 isolates

\begin{tabular}{|c|c|c|c|c|c|c|c|c|c|c|c|c|}
\hline \multirow[b]{3}{*}{ at $n t$} & \multicolumn{6}{|c|}{ Insertion } & \multicolumn{6}{|c|}{ Deletion } \\
\hline & \multicolumn{2}{|c|}{ Sample 9} & \multicolumn{2}{|c|}{ Sample 10} & \multicolumn{2}{|c|}{ Sample 13} & \multicolumn{2}{|c|}{ Sample 9} & \multicolumn{2}{|c|}{ Sample 10} & \multicolumn{2}{|c|}{ Sample 13} \\
\hline & S1 & S2 & S1 & S2 & S1 & S2 & S1 & S2 & S1 & S2 & S1 & S2 \\
\hline 2153 & $A$ & & & & & & & & & & & \\
\hline 2253 & & & $\mathrm{~T}$ & & & & & & & & & \\
\hline 2265 & A & & A & & & & & & & & & \\
\hline 3081 & & & & & & & & & $\mathrm{~T}$ & & & \\
\hline 3083 & $\mathrm{AA}$ & & & & & & & & & & & \\
\hline 3083 & & & & & & & & $A$ & & & & \\
\hline 3767 & & $\pi$ & & & & & & & & & & \\
\hline 4460 & & & & & CC & & & & & & & \\
\hline 5632 & & & & & & $\mathrm{~T}$ & & & & & & \\
\hline Total & 4 & 2 & 2 & 0 & 2 & 1 & 0 & 1 & 1 & 0 & 0 & 0 \\
\hline
\end{tabular}

Summarized in this table are nucleotide insertions/deletions introduced by long PCR amplification from the two individual clones in each of three HPV58 isolates. S1 and S2 denotes Sanger sequence \#1 (clone \#1) and \#2 (clone \#2), respectively. Numbers are nucleotide positions in the reference HPV58 genome at where the insertion or deletion was identified in each HPV58 isolate.

Zhejiang University. The study was approved by the Institutional Review Board for clinical research of this hospital. Informed consent was obtained from each participant prior the study. DNA was isolated from each sample by using TRIzol Reagent (Invitrogen, Carlsbad, CA) according to the manufacturer's instructions.

RCA enrichment, RCA chimeras debranching, paired-end library preparation and deep sequencing

Rolling circle amplification (RCA) based on phi29 DNA polymerase used to enrich the HPV58 genome and RCA chimeras debranching, paired-end library preparation and deep sequencing with an Illumina HiSeq-2000 platform have described in our other publications [8,31].

Table 3 Nucleotide substitutions by long-PCR amplification in HPV58 isolates create or inactivate restriction enzyme digestion sites

\begin{tabular}{lcccc}
\hline & & \multicolumn{3}{c}{ Sample number } \\
\cline { 3 - 5 } Enzyme & nt position* & $\mathbf{9}$ & $\mathbf{1 0}$ & $\mathbf{1 3}$ \\
\hline Afel & 1167 & + & - & - \\
Fspl & 2860 & - & - & + \\
Dralll (inactive) & 2925 & + & - & - \\
Fspl & 3940 & + & - & - \\
EcoRV & 5095 & - & + & - \\
BstXI & 5556 & + & - & - \\
Mscl (inactive) & 6352 & + & - & - \\
Eael & 6352 & + & - & -
\end{tabular}

Summarized in this table are distinguishable restriction enzyme cutting sites due to nucleotide substitutions introduced by long PCR amplification in three HPV58 isolates. See other details from Figure 3 legends. *The numbers are nucleotide positions with the substitutions to the reference HPV58 genome.
Long PCR template preparation, cloning, and sequencing RCA products prepared separately from three clinical samples (sample 9, 10 and 13) without S1 digestion and DNA shearing were selectively linearized by AgeI or DraII digestion and then amplified with a primer pair Pr3506 (5'-GACAGTAGACCACGAGGA-3', forward) and Pr7036 (5'-TACTCAGGATC/CGTCCCAAAGGAAACTG ATC-3', backward) for AgeI-digested RCA products, and with a primer pair Pr6906 (5'-TACATCGAATT/CTCCC AGGCTATTACTTGC-3', forward) and Pr3694 (5' -CCA ATGCCATGTGGATGAC-3', backward) for Drall-digested RCA products, respectively, using the Expand Long Template PCR System (Roche, Cat No. 11681834001). In brief, $2 \mu \mathrm{l}$ of digested RCA products were used for amplification in a 50- $\mu \mathrm{l}$ reaction together with $38.75 \mu \mathrm{l}$ DEPC-treated water, $2.5 \mu \mathrm{l} \mathrm{dNTP}$ mix (10 mM each)), $0.5 \mu \mathrm{l}$ of each forward and reverse primers $(20 \mu \mathrm{M}), 5 \mu \mathrm{L}$ of $10 \mathrm{x}$ PCR buffer II with $\mathrm{MgCl} 2$, and $0.75 \mu \mathrm{l}$ of the expand long template enzyme mix which contains high-fidelity thermostable Tgo DNA polymerase with proofreading activity. Individual long PCR product was gel-purified and cloned into pCR-XL-TOPO vector according to the manufacturer's instruction (Invitrogen, Cat No K4700-10). In brief, the PCR products generated from AgeI-digested RCA products using primer pairs Pr3506 (forward) and Pr7036 (backward) and the PCR products generated from DraII-digested RCA products using primer pairs Pr6906 (forward) and Pr3694 (backward) were precipitated, purified by agarose gel electrophoresis using crystal violet and then isolated using S.N.A.P Gel Purification Kit (Invitrogen). Purified long PCR products were cloned into a pCR-XL-TOPO vector. Two clones from each plasmid (Table 1) were prepared and sequenced from two different directions. Sequencing results 

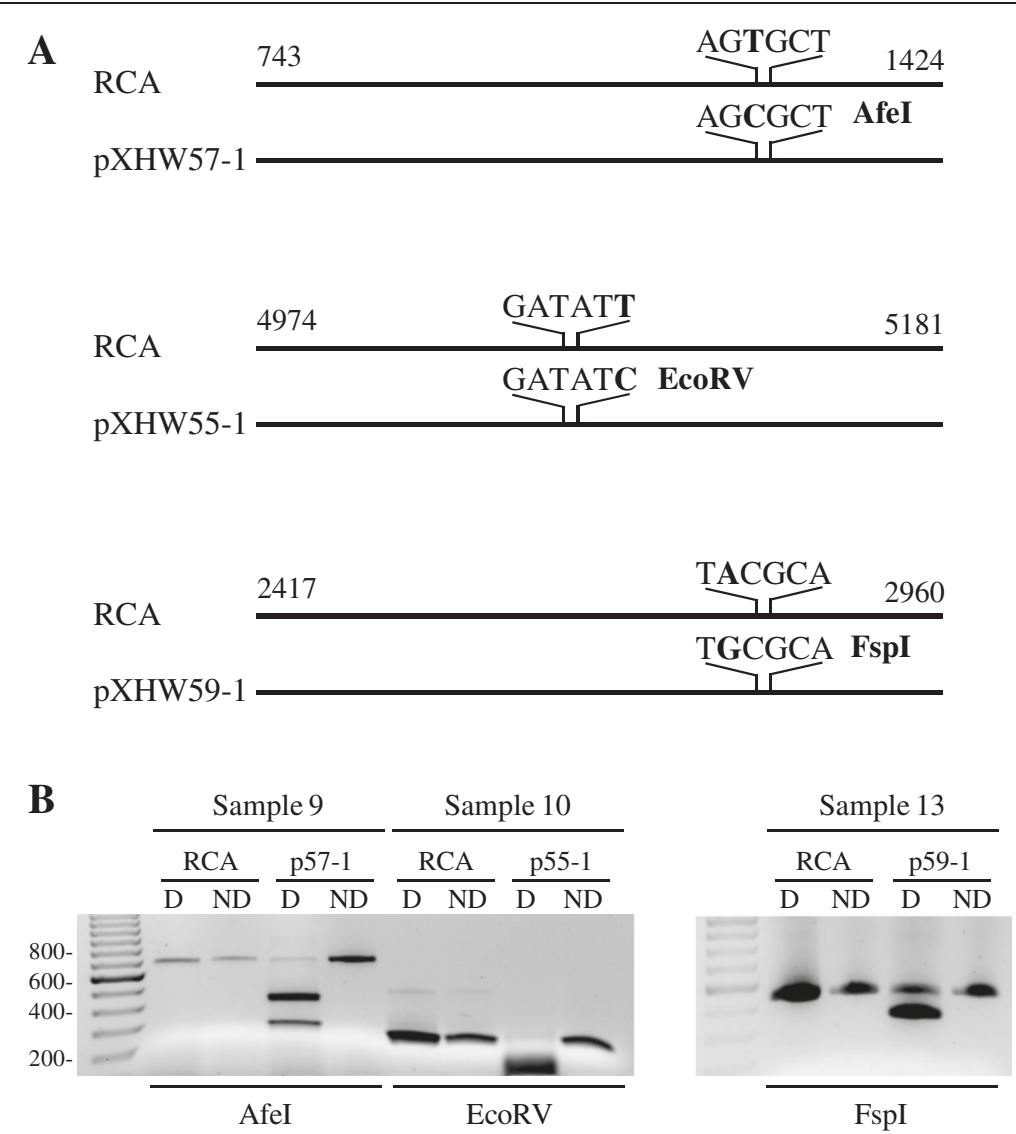

Figure 3 Restriction enzyme digestion distinguishes HPV58 amplicons from RCA products to their corresponding plasmids. (A) Diagrams of HPV58 amplicons from RCA samples and their corresponding plasmid clones with a new restriction enzyme cutting site. (B) Restriction enzyme digestion of RCA products and their corresponding plasmid clones from each clinical sample. RCA products and pXHW57-1 from the sample 9 were amplified using a primer pair of Pr743 and Pr1424 and digested with Afel. RCA products and pXHW55-1 from the sample 10 were amplified using a primer pair of Pr4974 and Pr5181 and digested with EcoRV. RCA products and pXHW59-1 from the sample 13 were amplified using a primer pair of Pr2417 and Pr2960 and digested with Fspl. The digested products were resolved in 1.5\% agarose gel. D: digested; ND: not digested.

of each clone were aligned against the HPV58 reference genome to identify nucleotide substitutions, insertions, or deletions.

\section{Restriction enzyme digestion}

RCA product and plasmid pXHW57-1 from the clinical sample 9 were amplified with primer pair Pr743 (5'-CGTGTTGTTACACTTGTGAC-3') and Pr1424 (5'-GTATTACAACTGTCTACATCCG-3'), and then digested with AfeI at $37^{\circ} \mathrm{C}$ overnight. RCA product and pXHW55-1 from the sample 10 were amplified with primer pair Pr4974 (5'-CATCTCCTCATAGACTTG TAAC-3') and Pr5181 (5' ${ }^{\prime}$-CGAGTACGAAGTGTAG CCT-3'), and then digested with EcoRV at $37^{\circ} \mathrm{C}$ overnight. RCA product and pXHW59-1 from the sample 13 were amplified with primer pair Pr2417 (5'-GTATGATA GATGATGTAACAGC-3') and Pr2960 (5'-ACGCTTTA GTCTTTGATGCTA-3'), and then digested with FspI at $37^{\circ} \mathrm{C}$ overnight. Non-digested products without enzymes were used as controls.

\section{Sequencing data analyses}

The trimmed reads were aligned to human (hg19 assembly) from UCSC genome browser and HPV58 reference genome (GenBank accession number D90400 or GI:222386) from PaVe database simultaneously using the Burrows-Wheeler Alignment tool (BWA) with default setting [42]. The output alignment files in SAM format were further processed using SAMtools [43]. The genome coverage files (WIGGLE or BAM files) were loaded onto the Integrative Genomics Viewer (IGV, www.broadinstitute.org/igv) to visualize sequence alignments, genomic annotations and substitutions [44] with defaulted settings. The cloning sequences were aligned to HPV58 reference genome using NCBI Blast tool.

\section{Quantitative real-time PCR}

Quantitative real-time PCR, with an HPV58-specific primer pair Pr 743 (5'-CGTGTTGTTACACTTGTG AC-3') and Pr 854 (5' ${ }^{\prime}$-CTAGGGCACACAATGGTA 
CA-3') using Power SYBR Green PCR Master Mix (Invitrogen) for high sensitivity and reproducibility, was performed on $100 \mathrm{pg}$ of samples DNA (sample \#10 and \#13) before and after RCA enrichment, of which the RCA products from clinical samples \#10 and \#13 without S1 digestion and DNA shearing were selectively linearized by AgeI or DraII digestion. A 10-fold serial dilution starting from $100 \mathrm{pg}\left(\sim 1.3 \times 10^{7}\right.$ copies $)$ of the plasmid pXW59 which contains an HPV58 DNA fragment from nt 6906 to 3695 was amplified using the same primer set by qPCR to create a standard curve. The threshold cycle $(\mathrm{Ct})$ values of qPCR data from 2 repeats were calculated for copy number analysis. A primer pair optimized for routine SYBR Green real-time PCR assays which specifically amplifies a genomic region containing human GAPDH promoter was purchased from Diagenode (Denville, NJ) and was used an internal control.

\section{Competing interests}

The authors declare that they have no competing interests.

\section{Authors' contributions}

All authors conceived, designed and analyzed the study. XW, YL and TN performed experiments, with $Y L$ and TN on RCA-seq and XW on long PCR template cloning and sequencing and all other assays. $Y L$ and $X X$ collected clinical samples and performed HPV screening. XW, YL and TN wrote the draft manuscript. ZMZ, JZ and XX finalized the manuscript. All authors read and approve the final manuscript.

\section{Acknowledgment}

This study was supported by the Intramural Research Programs of the NCl, Center for Cancer Research and the NHLBI, NIH, and the Natural Science Foundation of China (NSFC 81172475) and the Natural Science Foundation of Zhejiang Province of China (grant NO: LQ13H160003).

\section{Author details}

'Tumor Virus RNA Biology Section, Gene Regulation and Chromosome Biology Laboratory, Center for Cancer Research, National Cancer Institute, National Institutes of Health, Bethesda, MD, USA. ${ }^{2}$ Department of Gynecologic Oncology, Women's Hospital, School of Medicine, Zhejiang University, Hangzhou, China. ${ }^{3}$ DNA Sequencing and Computational Biology Core, National Heart Lung and Blood Institute, National Institutes of Health, Bethesda, MD, USA. ${ }^{4}$ State Key Laboratory of Genetics Engineering and Ministry of Education Key Laboratory of Contemporary Anthropology, School of Life Sciences, Fudan University, Shanghai, China.

Received: 2 September 2013 Accepted: 16 December 2013 Published: 13 January 2014

\section{References}

1. Munoz N, Bosch FX, de Sanjose S, Herrero R, Castellsague X, Shah KV, Snijders PJ, Meijer CJ: Epidemiologic classification of human papillomavirus types associated with cervical cancer. N Engl J Med 2003, 348:518-527.

2. Walboomers JM, Jacobs MV, Manos MM, Bosch FX, Kummer JA, Shah KV, Snijders PJ, Peto J, Meijer CJ, Munoz N: Human papillomavirus is a necessary cause of invasive cervical cancer worldwide. J Pathol 1999, 189:12-19.

3. Durst M, Gissmann L, Ikenberg H, Zur HH: A papillomavirus DNA from a cervical carcinoma and its prevalence in cancer biopsy samples from different geographic regions. Proc Natl Acad Sci USA 1983, 80:3812-3815.

4. Zur Hausen H: Papillomaviruses and cancer: from basic studies to clinical application. Nat Rev Cancer 2002, 2:342-350.

5. Hong D, Ye F, Chen H, Lu W, Cheng Q, Hu Y, Xie X: Distribution of human papillomavirus genotypes in the patients with cervical carcinoma and its precursors in Zhejiang Province, China. Int J Gynecol Cancer 2008, 18:104-109.

6. Chan PK, Luk AC, Park JS, Smith-McCune KK, Palefsky JM, Konno R, Giovannelli L, Coutlee F, Hibbitts S, Chu TY, Settheetham-Ishida W, Picconi MA, Ferrera A, De MF, Woo YL, Raiol T, Pina-Sanchez P, Cheung JL, Bae JH, Chirenje MZ, Magure T, Moscicki AB, Fiander AN, Di SR, Cheung TH, Yu MM, Tsui SK, Pim D, Banks L: Identification of human papillomavirus type 58 lineages and the distribution worldwide. J Infect Dis 2011, 203:1565-1573.

7. Chan PK: Human papillomavirus type 58: the unique role in cervical cancers in East Asia. Cell Biosci 2012, 2:17.

8. Li Y, Wang X, Ni T, Wang F, Lu W, Zhu J, Xie X, Zheng ZM: Human papillomavirus type 58 genome variations and RNA expression in cervical lesions. J Virol 2013, 87:9313-9322.

9. Zheng ZM, Baker CC: Papillomavirus genome structure, expression, and post-transcriptional regulation. Front Biosci 2006, 11:2286-2302.

10. Bernard HU, Burk RD, Chen Z, Van DK, Zur HH, De Villiers EM: Classification of papillomaviruses (PVs) based on 189 PV types and proposal of taxonomic amendments. Virology 2010, 401:70-79.

11. De Villiers EM, Fauquet C, Broker TR, Bernard HU, Zur HH: Classification of papillomaviruses. Virology 2004, 324:17-27.

12. Bernard HU, Calleja-Macias IE, Dunn ST: Genome variation of human papillomavirus types: phylogenetic and medical implications. Int I Cancer 2006, 118:1071-1076.

13. Ho L, Chan SY, Burk RD, Das BC, Fujinaga K, Icenogle JP, Kahn T, Kiviat N, Lancaster W, Mavromara-Nazos P: The genetic drift of human papillomavirus type 16 is a means of reconstructing prehistoric viral spread and the movement of ancient human populations. J Virol 1993, 67:6413-6423.

14. Cornet I, Gheit T, Franceschi S, Vignat J, Burk RD, Sylla BS, Tommasino M, Clifford GM: Human papillomavirus type 16 genetic variants: phylogeny and classification based on E6 and LCR. J Virol 2012, 86:6855-6861.

15. Arias-Pulido H, Peyton CL, Torrez-Martinez N, Anderson DN, Wheeler CM: Human papillomavirus type 18 variant lineages in United States populations characterized by sequence analysis of LCR-E6, E2, and L1 regions. Virology 2005, 338:22-34.

16. Yamada T, Manos MM, Peto J, Greer CE, Munoz N, Bosch FX, Wheeler CM: Human papillomavirus type 16 sequence variation in cervical cancers: a worldwide perspective. J Virol 1997, 71:2463-2472.

17. Chan SY, Delius H, Halpern AL, Bernard HU: Analysis of genomic sequences of 95 papillomavirus types: uniting typing, phylogeny, and taxonomy. J Virol 1995, 69:3074-3083.

18. Pande S, Jain N, Prusty BK, Bhambhani S, Gupta S, Sharma R, Batra S, Das BC: Human papillomavirus type 16 variant analysis of E6, E7, and L1 genes and long control region in biopsy samples from cervical cancer patients in north India. J Clin Microbiol 2008, 46:1060-1066.

19. Shang Q, Wang Y, Fang Y, Wei L, Chen S, Sun Y, Li B, Zhang F, Gu H: Human papillomavirus type 16 variant analysis of E6, E7, and L1 [corrected] genes and long control region in [corrected] cervical carcinomas in patients in northeast China. J Clin Microbiol 2011, 49:2656-2663.

20. Sun $M$, Gao L, Liu Y, Zhao Y, Wang X, Pan Y, Ning T, Cai H, Yang H, Zhai W, Ke $Y$ : Whole genome sequencing and evolutionary analysis of human papillomavirus type 16 in central China. PLOS ONE 2012, 7:e36577.

21. Arroyo SL, Basaras M, Arrese E, Hernaez S, Andia D, Esteban V, Garcia-Etxebarria K, Jugo BM, Cisterna R: Human papillomavirus (HPV) genotype 18 variants in patients with clinical manifestations of HPV related infections in Bilbao, Spain. Virol J 2012, 9:258.

22. Sun Z, Liu J, Wang G, Zhou W, Liu C, Ruan Q: Variant lineages of human papillomavirus type 18 in Northeast China populations characterized by sequence analysis of E6, E7, and L1 regions. Int J Gynecol Cancer 2012, 22:930-936

23. Xi LF, Koutsky LA, Galloway DA, Kuypers J, Hughes JP, Wheeler CM, Holmes KK, Kiviat NB: Genomic variation of human papillomavirus type 16 and risk for high grade cervical intraepithelial neoplasia. J Nat/ Cancer Inst 1997, 89:796-802.

24. Zuna RE, Moore WE, Shanesmith RP, Dunn ST, Wang SS, Schiffman M, Blakey GL, Teel T: Association of HPV16 E6 variants with diagnostic severity in cervical cytology samples of 354 women in a US population. Int J Cancer 2009, 125:2609-2613.

25. Gheit T, Cornet I, Clifford GM, Iftner T, Munk C, Tommasino M, Kjaer SK: Risks for persistence and progression by human papillomavirus type 16 
variant lineages among a population-based sample of Danish women. Cancer Epidemiol Biomarkers Prev 2011, 20:1315-1321.

26. Niccoli S, Abraham S, Richard C, Zehbe I: The Asian-American E6 variant protein of human papillomavirus 16 alone is sufficient to promote immortalization, transformation, and migration of primary human foreskin keratinocytes. J Virol 2012, 86:12384-12396.

27. Bzhalava D, Johansson H, Ekstrom J, Faust H, Moller B, Eklund C, Nordin P, Stenquist B, Paoli J, Persson B, Forslund O, Dillner J: Unbiased approach for virus detection in skin lesions. PLOS ONE 2013, 8:e65953.

28. Johansson H, Bzhalava D, Ekstrom J, Hultin E, Dillner J, Forslund O: Metagenomic sequencing of "HPV-negative" condylomas detects novel putative HPV types. Virology 2013, 440:1-7.

29. Meiring TL, Salimo AT, Coetzee B, Maree HJ, Moodley J, Hitzeroth II, Freeborough MJ, Rybicki EP, Williamson AL: Next-generation sequencing of cervical DNA detects human papillomavirus types not detected by commercial kits. Virol J 2012, 9:164.

30. Marincevic-Zuniga Y, Gustavsson I, Gyllensten U: Multiply-primed rolling circle amplification of human papillomavirus using sequence-specific primers. Virology 2012, 432:57-62.

31. Ni T, Corcoran DL, Rach EA, Song S, Spana EP, Gao Y, Ohler U, Zhu J: A paired-end sequencing strategy to map the complex landscape of transcription initiation. Nat Methods 2010, 7:521-527.

32. Kirii Y, Iwamoto S, Matsukura T: Human papillomavirus type 58 DNA sequence. Virology 1991, 185:424-427.

33. Johne R, Muller H, Rector A, Van RM, Stevens H: Rolling-circle amplification of viral DNA genomes using phi29 polymerase. Trends Microbiol 2009, 17:205-211.

34. Rector A, Tachezy R, Van RM: A sequence-independent strategy for detection and cloning of circular DNA virus genomes by using multiply primed rolling-circle amplification. J Virol 2004, 78:4993-4998.

35. Dean FB, Nelson JR, Giesler TL, Lasken RS: Rapid amplification of plasmid and phage DNA using Phi 29 DNA polymerase and multiply-primed rolling circle amplification. Genome Res 2001, 11:1095-1099.

36. Esteban JA, Salas M, Blanco L: Fidelity of phi 29 DNA polymerase. Comparison between protein-primed initiation and DNA polymerization. J Biol Chem 1993, 268:2719-2726.

37. Quail MA, Smith M, Coupland P, Otto TD, Harris SR, Connor TR, Bertoni A, Swerdlow HP, Gu Y: A tale of three next generation sequencing platforms: comparison of lon Torrent. Pacific Biosciences and Illumina MiSeq sequencers. BMC Genomics 2012, 13:341.

38. Eckert KA, Kunkel TA: DNA polymerase fidelity and the polymerase chain reaction. PCR Methods Appl 1991, 1:17-24.

39. Saiki RK, Gelfand DH, Stoffel S, Scharf SJ, Higuchi R, Horn GT, Mullis KB, Erlich HA: Primer-directed enzymatic amplification of DNA with a thermostable DNA polymerase. Science 1988, 239:487-491.

40. Shao W, Boltz VF, Spindler JE, Kearney MF, Maldarelli F, Mellors JW, Stewart C, Volfovsky N, Levitsky A, Stephens RM, Coffin JM: Analysis of 454 sequencing error rate, error sources, and artifact recombination for detection of Low-frequency drug resistance mutations in HIV-1 DNA. Retrovirology 2013, 10:18.

41. Brodin J, Mild M, Hedskog C, Sherwood E, Leitner T, Andersson B, Albert J: PCR-Induced Transitions Are the Major Source of Error in Cleaned Ultra-Deep Pyrosequencing Data. PLoS ONE 2013, 8:e70388.

42. Li H, Durbin R: Fast and accurate short read alignment with BurrowsWheeler transform. Bioinformatics 2009, 25:1754-1760.

43. Li H, Handsaker B, Wysoker A, Fennell T, Ruan J, Homer N, Marth G, Abecasis G, Durbin R: The Sequence Alignment/Map format and SAMtools. Bioinformatics 2009, 25:2078-2079.

44. Robinson JT, Thorvaldsdottir H, Winckler W, Guttman M, Lander ES, Getz G, Mesirov JP: Integrative genomics viewer. Nat Biotechnol 2011, 29:24-26.

doi:10.1186/2045-3701-4-5

Cite this article as: Wang et al:: Genome sequencing accuracy by RCA-seq versus long PCR template cloning and sequencing in identification of human papillomavirus type 58. Cell \& Bioscience 2014 4:5.

\section{Submit your next manuscript to BioMed Central and take full advantage of:}

- Convenient online submission

- Thorough peer review

- No space constraints or color figure charges

- Immediate publication on acceptance

- Inclusion in PubMed, CAS, Scopus and Google Scholar

- Research which is freely available for redistribution

Submit your manuscript at www.biomedcentral.com/submit
C Biomed Central 